\title{
Maintenance of the paraspinal muscles may protect against radiographic knee osteoarthritis
}

This article was published in the following Dove Press journal:

Open Access Rheumatology: Research and Reviews

10 August 2017

Number of times this article has been viewed

\author{
Koichiro Azuma' \\ Yasushi Sera' \\ Takuma Shinjo' \\ Michiyo Takayama \\ Eisuke Shiomi \\ Suketaka Momoshima \\ Yasushi Iwao ${ }^{2}$ \\ Hiroyuki Ishida ${ }^{3}$ \\ Hideo Matsumoto' \\ 'Institute for Integrated Sports \\ Medicine, Keio University School \\ of Medicine, ${ }^{2}$ Center for Preventive \\ Medicine, Keio University Hospital, \\ Shinjuku-ku, Tokyo, ${ }^{3}$ Sports \\ Medicine Research Center, Keio \\ University, Kohoku-ku, Yokohama, \\ Kanagawa, Japan
}

Background: Knee osteoarthritis (OA) is an increasing health problem worldwide. So far, only obesity and quadriceps weakness are identified as modifiable risk factors for knee OA. Core muscle strengthening is becoming increasingly popular among older adults because of its ability to enhance the activities of daily living during old age. This study investigated the associations of the size and quality of the abdominal trunk muscles with radiographic knee osteoarthritis (RKOA).

Methods: From 2012 to 2016, data were collected from 146 males and 135 females (age $63.9 \pm 13.4$ years, BMI $23.2 \pm 3.8 \mathrm{~kg} / \mathrm{m}^{2}$ ) at annual musculoskeletal examinations, including knee radiographs and body composition analyses, by dual-energy X-ray absorptiometry. Crosssectional areas of abdominal trunk muscles were measured using a single-slice computed tomography scan image obtained at the level of the umbilicus.

Results: The prevalence of RKOA was $21.2 \%$ in males and $28.1 \%$ in females. Compared to subjects without RKOA, subjects with RKOA were $\sim 6$ years older and had smaller paraspinal muscle $\left(38.4 \pm 8.7\right.$ vs $33.1 \pm 10.1 \mathrm{~cm}^{2}, p<0.01$ in males; $24.1 \pm 7.1$ vs $20.7 \pm 7.5 \mathrm{~cm}^{2}, p<0.05$ in females). In contrast, there was no decrease in appendicular or total lean mass, and only in females, BMI and total fat mass (FM) were higher in subjects with RKOA $(21.5 \pm 3.5 \mathrm{vs}$ $24.5 \pm 4.4 \mathrm{~kg} / \mathrm{m}^{2}, 16.7 \pm 7.0 \mathrm{vs} 20.5 \pm 7.7 \mathrm{~kg}$, respectively, both $\left.p<0.01\right)$. After adjusting for age and sex, smaller cross-sectional area/lower attenuation value of the paraspinal muscles was associated with RKOA (both $p<0.05$ ), while greater appendicular or total lean mass as well as greater FM was associated with RKOA. The size and quality of the paraspinal muscles were not associated with knee pain or habitual exercise.

Conclusion: Small, poor-quality paraspinal muscles may be linked to a higher risk of RKOA, but appendicular or total lean mass was not a good predictor of RKOA.

Keywords: radiographic knee osteoarthritis, paraspinal muscle cross sectional area, paraspinal muscle attenuation, lean body mass, fat mass

\section{Introduction}

The prevalence of knee osteoarthritis (OA), which is characterized by narrowing of the joint space and osteophyte formation, is an increasing problem worldwide. ${ }^{1}$ In Japan, radiographic knee OA is estimated to affect $25,300,000$ people over 40 years of age, ${ }^{2}$ and a recent national survey ranked OA fourth among disabling diseases that cause affected people to require support with their activities of daily living.

The etiology of knee OA is complex, and several risk factors, including obesity, previous injury, female sex, advancing age, and quadriceps weakness, have been identified. ${ }^{3}$ Of these risk factors, only obesity and muscular strength are modifiable. 
Therefore, weight loss and quadriceps resistance training are frequently recommended to alleviate knee pain and slow the progression of knee OA. However, for individuals with knee OA, weight loss through aerobic exercise (such as walking) and quadriceps training are both made difficult by pain during movement.

Core muscle strengthening exercises are becoming increasingly popular among older adults because these exercises enhance their ability to successfully perform the activities of daily living during old age. ${ }^{4}$ In fact, it has been demonstrated that trunk muscle activity occurs before the activity of the prime mover of the lower limb, ${ }^{5}$ and diminished core stability due to impaired abdominal trunk muscle is associated with lower extremity injury, including the knee. ${ }^{6}$ Improved core strength may also correct dynamic malalignment of the lower extremity by reducing the knee abduction moment. ${ }^{7}$ Thus, the objective of this study was to examine the association of abdominal trunk muscle size and quality with radiographic knee OA, independent of BMI or whole body/appendicular lean mass.

\section{Methods}

\section{Study subjects}

A total of 281 older adults who had undergone knee radiographs and dual-energy X-ray absorptiometry (DEXA) in the Center for Preventive Medicine of Keio University Hospital between September 2012 and March 2016 as part of screening for early signs of musculoskeletal degeneration were enrolled in this study. We obtained information on age, sex, body mass index (BMI; $\mathrm{kg} / \mathrm{m}^{2}$ ), knee pain, and exercise habits from questionnaires. Habitual exercise was defined as exercising for at least 30 minutes per session, at least twice per week, for at least 1 year, according to the National Health and Nutrition Survey conducted by the Ministry of Health, Labour and Welfare in Japan.

Because this was a nonintrusive retrospective study, informed consent was not obtained from the study subjects. All records were anonymized and de-identified prior to analysis. This study was approved by the local ethics committee of the Keio University School of Medicine (No. 20140323).

\section{Imaging and measurements}

\section{Knee radiographs}

Anteroposterior and lateral radiographs of bilateral knees were taken in a supine position at Keio University Hospital. The radiographs were read by a single, experienced knee surgeon, and Kellgren/Lawrence (K/L) grades were assigned as described in the $\mathrm{K} / \mathrm{L}$ radiographic atlas. ${ }^{8}$ In the $\mathrm{K} / \mathrm{L}$ grading system, radiographs are scored from 0 to 4 , with higher grades being associated with more severe OA. The definition of $\mathrm{K} / \mathrm{L}$ grade itself is, however, rather qualitative, especially for joint space narrowing. ${ }^{9,10}$ Unfortunately, our radiographs were taken in the non-weight-bearing position, but the $\mathrm{K} / \mathrm{L}$ grade $\geq 2$ under this condition is at least $\geq 2$ if taken in a weight-bearing position. It is also obvious for experienced knee surgeons to determine the difference between $\mathrm{K} / \mathrm{L}$ grade 1 (doubtful narrowing of joint space and possible osteophytic lipping) and grade 2 (definite osteophytes and possible narrowing of joint space). Therefore, in the current study, subjects with $\mathrm{K} / \mathrm{L}$ grades $\geq 2$ for at least one knee were considered to have radiographic knee osteoarthritis (RKOA).

\section{DEXA scan}

Whole-body DEXA scans (Lunar Prodigy® Advance; GE Healthcare Japan Corporation, Tokyo, Japan) were performed using enCORE software, version 9.2 (GE Healthcare). Standard scan mode was used for whole-body scans to measure total and regional (upper body, trunk, and legs) fat mass (FM) and lean body mass (LBM). Appendicular skeletal muscle mass (ASM) was defined as the LBM of both the upper and lower extremities. Skeletal muscle index (SMI) was defined as the ASM divided by height squared. ${ }^{11}$

\section{Computed tomography (CT) scan}

Abdominal trunk muscle and visceral adipose tissue (VAT) were measured using a single-slice CT image obtained at the level of the umbilicus with $5 \mathrm{~mm}$ slice thickness. ${ }^{12}$

The images were exported to a workstation in a standard DICOM (Digital Imaging and Communications in Medicine) format and were analyzed using sliceOmatic software (TomoVision, Magog, QC, Canada). The cross-sectional areas of psoas major, the paraspinal muscles (multifidus, longissimus, and iliocostalis lumborum), and the anterolateral abdominal muscles (Ant abd; the internal and external oblique muscles and transversus abdominis), as well as VAT, were measured by defining a range of $\mathrm{CT}$ attenuation values and a region of interest for each tissue. The attenuation values were -30 to $-190 \mathrm{HU}$ for VAT and 0-100 HU for muscle, as previously described. ${ }^{13}$ Low muscle attenuation (a low CT attenuation value for muscle) indicates increased muscle fat content ${ }^{13}$ and has been reported to be a marker of poor muscle quality and muscle weakness independent of age-associated loss in muscle mass $;{ }^{14}$ therefore, muscle attenuation was assessed simultaneously with cross-sectional area. 


\section{Statistical analysis}

The significance of between-group differences (with and without RKOA) was assessed using analysis of variance (ANOVA). Homogeneity of variances was tested by Levene's test, and if the assumption was violated, Welch's test was used. Multivariable linear regression models were used to adjust for age, sex, and BMI or LBM/FM. Variance inflation factor (VIF) was used to check for multiple collinearity. Two-way ANOVA was also used to assess the interaction between RKOA and sex. Separate stepwise-regression analyses for RKOA were performed for males and females. IBM SPSS for Windows, Version 23 (IBM Corporation, Armonk, NY, USA) was used for all statistical analyses. The significance level was set at $p<0.05$, and all values are presented as mean \pm standard deviation (SD).

\section{Results}

\section{Characteristics of the subjects}

As shown in Table 1, subjects with radiographic knee osteoarthritis (RKOA) were older than those without RKOA; this was true for both males and females. In both sexes, BMI data showed a significant interaction between RKOA and sex $(p<0.05)$. In female subjects only, RKOA was associated with a higher BMI and greater FM. Although there was no other interaction between RKOA and sex, except for BMI, we have shown the data by sex, due to its large difference in body composition.
Analysis of the CT images showed that paraspinal muscle cross-sectional area was significantly lower among both males and females with RKOA, while paraspinal muscle CT attenuation was lower only in females with RKOA. In addition, female subjects with RKOA had more visceral adiposity and greater total adiposity than those without RKOA.

\section{Multivariable analyses}

Table 2 shows the results of multivariable linear regression models of body composition in subjects with and without RKOA, in which the subjects without RKOA serve as the referent group. After adjusting for sex and age (model 2), those with RKOA were heavier and had more LBM and FM than those without RKOA. In contrast, subjects with RKOA had smaller paraspinal muscle area and lower paraspinal muscle attenuation on $\mathrm{CT}$, with concomitantly larger psoas muscle area, than those without RKOA. After adding BMI to the model (model 3), only the between-group difference in paraspinal muscle area remained significant. Surprisingly, after adjusting for age, sex, and BMI, lower FM was positively associated with RKOA. After adjusting for age, sex, height, and FM (model 4), greater total and appendicular LBM were more prominently associated with RKOA. After adjusting for age, sex, height, and LBM (model 5), the associations of smaller Ant abd and paraspinal muscle area with RKOA became more robust, while the association between greater FM and RKOA became insignificant.

Table I Body composition characteristics of the study subjects stratified using the $\mathrm{K} / \mathrm{L}$ grading system for RKOA

\begin{tabular}{|c|c|c|c|c|}
\hline Characteristics & Male & & Female & \\
\hline RKOA & $0-I(n=\mid 15)$ & $>2(n=3 I)$ & $0-I(n=97)$ & $>2(n=38)$ \\
\hline Age & $60.8 \pm 15.4$ & $67.3 \pm 12.3^{*}$ & $63.8 \pm 11.6$ & $71.0 \pm 8.6 * *$ \\
\hline Height (cm) & $168.8 \pm 6.6$ & $167.0 \pm 7.2$ & $156.0 \pm 6.7$ & $151.8 \pm 6.3 * *$ \\
\hline BW (kg) & $67.7 \pm 11.2$ & $69.1 \pm 11.5$ & $52.3 \pm 9.8$ & $56.5 \pm 10.9 *$ \\
\hline BMI $\left(\mathrm{kg} / \mathrm{m}^{2}\right)$ & $23.7 \pm 3.3$ & $24.7 \pm 3.4$ & $21.5 \pm 3.5$ & $24.5 \pm 4.4 * *$ \\
\hline \multicolumn{5}{|l|}{ DEXA } \\
\hline$\%$ body fat & $24.4 \pm 7.2$ & $25.3 \pm 6.6$ & $30.9 \pm 7.8$ & $35.2 \pm 7.2 * *$ \\
\hline $\mathrm{FM}(\mathrm{kg})$ & $|7| \pm 7.5$. & $17.9 \pm 6.9$ & $16.7 \pm 7.0$ & $20.5 \pm 7.7 * *$ \\
\hline LBM (kg) & $50.6 \pm 5.7$ & $51.2 \pm 6.7$ & $35.6 \pm 4.5$ & $36.0 \pm 4.4$ \\
\hline SMI & $7.3 \pm 0.8$ & $7.5 \pm 0.9$ & $5.7 \pm 0.6$ & $6.1 \pm 0.7^{* *}$ \\
\hline \multicolumn{5}{|l|}{ CT at umbilical level } \\
\hline Psoas $\left(\mathrm{cm}^{2}\right)$ & $23.0 \pm 5.8$ & $23.7 \pm 4.3$ & $13.2 \pm 3.6$ & $12.9 \pm 3.5$ \\
\hline Psoas HU & $49.2 \pm 4.2$ & $48.8 \pm 4.2$ & $45.7 \pm 4.2$ & $44.3 \pm 4.1$ \\
\hline Paraspinal $\left(\mathrm{cm}^{2}\right)$ & $38.4 \pm 8.7$ & $33.1 \pm 10.1 * *$ & $24.1 \pm 7.1$ & $20.7 \pm 7.5^{*}$ \\
\hline Paraspinal HU & $47.3 \pm 6.5$ & $44.5 \pm 7.1^{*}$ & $39.4 \pm 7.0$ & $34.6 \pm 5.6 * *$ \\
\hline Ant abd $\left(\mathrm{cm}^{2}\right)$ & $49.8 \pm 10.5$ & $47.2 \pm 13.9$ & $27.8 \pm 8.1$ & $25.8 \pm 8.6$ \\
\hline Ant abd HU & $44.0 \pm 6.8$ & $42.3 \pm 6.2$ & $38.9 \pm 7.6$ & $34.7 \pm 4.9 * *$ \\
\hline $\operatorname{VAT}\left(\mathrm{cm}^{2}\right)$ & $100.8 \pm 53.1$ & $114.1 \pm 49.9$ & $64.4 \pm 39.1$ & $82.3 \pm 43.6 *$ \\
\hline
\end{tabular}

Notes: $* p<0.05$ and $* * p<0.01$ vs subjects without RKOA, by ANOVA. Welch's test was used for Ant abd HU in females, since the assumption of homogeneity of variances was violated. Data presented as mean \pm SD unless otherwise indicated.

Abbreviations: ANOVA, analysis of variance; Ant abd, anterolateral abdominal muscle; BMI, body mass index; BW, body weight; CT, computed tomography; DEXA, dualenergy X-ray absorptiometry; FM, fat mass; HU, Hounsfield unit; K/L, Kellgren/Lawrence; LBM, lean body mass; Paraspinal, paraspinal muscle; Psoas, psoas major muscle; RKOA, radiographic knee osteoarthritis; SMI, skeletal muscle index; VAT, visceral adipose tissue. 
Table 2 Multivariable analysis of body composition and trunk muscle characteristics in subjects with and without RKOA

\begin{tabular}{|c|c|c|c|c|c|}
\hline Model & I & 2 & 3 & 4 & 5 \\
\hline \multicolumn{6}{|l|}{ All $(n=28 I)$} \\
\hline BMI $\left(\mathrm{kg} / \mathrm{m}^{2}\right)$ & $0.22 * * *$ & $0.27^{* * *}$ & - & $0.58 * * *$ & 0.16 \\
\hline $\mathrm{FM}(\mathrm{kg})$ & $0.14 *$ & $0.16 * *$ & $-0.35^{* *}$ & - & 0.09 \\
\hline LBM (kg) & -0.04 & $0.27^{*}$ & 0.01 & $0.47 * *$ & - \\
\hline ASM (kg) & -0.05 & $0.29 *$ & 0.08 & $0.48 * * *$ & - \\
\hline Psoas $\left(\mathrm{cm}^{2}\right)$ & -0.05 & $0.27^{* *}$ & 0.11 & $0.24^{*}$ & 0.08 \\
\hline Psoas HU & $-0.12^{*}$ & 0.04 & 0.07 & 0.08 & 0.02 \\
\hline Paraspinal $\left(\mathrm{cm}^{2}\right)$ & $-0.22 * * *$ & $-0.19 *$ & $-0.27^{* *}$ & -0.18 & $-0.35 * *$ \\
\hline Paraspinal HU & $-0.25 * * *$ & $-0.21 *$ & -0.13 & -0.15 & $-0.17 *$ \\
\hline Ant abd $\left(\mathrm{cm}^{2}\right)$ & $-0.12 *$ & 0 & $-0.20 *$ & -0.03 & $-0.38 * *$ \\
\hline Ant abd HU & $-0.20 * *$ & -0.1 & 0 & -0.02 & -0.09 \\
\hline \multicolumn{6}{|l|}{ Male $(n=\mid 46)$} \\
\hline BMI $\left(\mathrm{kg} / \mathrm{m}^{2}\right)$ & 0.12 & 0.16 & - & $0.43^{*}$ & 0.04 \\
\hline $\mathrm{FM}(\mathrm{kg})$ & 0.05 & 0.07 & -0.25 & - & 0.02 \\
\hline LBM (kg) & 0.04 & 0.15 & 0.07 & $0.25 *$ & - \\
\hline ASM (kg) & 0.02 & 0.15 & 0.08 & $0.26 *$ & - \\
\hline Psoas $\left(\mathrm{cm}^{2}\right)$ & 0.05 & $0.22 *$ & 0.17 & $0.22 *$ & 0.14 \\
\hline Psoas HU & -0.04 & 0.09 & 0.12 & 0.12 & 0.07 \\
\hline Paraspinal $\left(\mathrm{cm}^{2}\right)$ & $-0.24 * *$ & $-0.20 *$ & $-0.23^{*}$ & -0.20 & $-0.35 * *$ \\
\hline Paraspinal HU & -0.17 & -0.11 & -0.05 & -0.08 & -0.09 \\
\hline Ant abd $\left(\mathrm{cm}^{2}\right)$ & -0.09 & -0.03 & -0.11 & -0.03 & $-0.26 *$ \\
\hline Ant abd HU & -0.11 & -0.02 & 0.05 & 0.03 & -0.02 \\
\hline \multicolumn{6}{|l|}{ Female $(n=\mid 35)$} \\
\hline BMI $\left(\mathrm{kg} / \mathrm{m}^{2}\right)$ & $0.35^{* * *}$ & $0.33^{* * *}$ & - & - & 0.22 \\
\hline $\mathrm{FM}(\mathrm{kg})$ & $0.23 * *$ & $0.25 * *$ & $-0.48 *$ & - & 0.14 \\
\hline LBM (kg) & 0.04 & 0.16 & -0.04 & $0.29 *$ & - \\
\hline ASM (kg) & 0.05 & $0.18^{*}$ & 0.02 & $0.30 * *$ & - \\
\hline Psoas $\left(\mathrm{cm}^{2}\right)$ & -0.04 & 0.12 & -0.05 & 0.07 & -0.09 \\
\hline Psoas HU & -0.15 & 0 & 0.02 & 0.04 & -0.03 \\
\hline Paraspinal $\left(\mathrm{cm}^{2}\right)$ & $-0.21 *$ & -0.05 & -0.17 & -0.11 & -0.15 \\
\hline Paraspinal HU & $-0.3 I^{* * *}$ & $-0.23 *$ & -0.16 & -0.18 & -0.20 \\
\hline Ant abd $\left(\mathrm{cm}^{2}\right)$ & -0.11 & 0.05 & -0.17 & -0.02 & -0.24 \\
\hline Ant abd HU & $-0.27 * *$ & -0.14 & -0.04 & -0.04 & -0.13 \\
\hline
\end{tabular}

Notes: Values in columns represent standardized beta coefficients from linear regression model. I: unadjusted; 2: adjusted for age and sex; 3: adjusted for age, sex, and BMI; 4: adjusted for age, sex, height, and FM; and 5: adjusted for age, sex, height, and LBM. ${ }^{*} p<0.05,{ }^{* *} p<0.0 \mathrm{I}$, and ${ }^{* * *} p<0.00 \mathrm{I}$, using multivariable linear regression models; subjects without radiographic knee OA are the referent group.

Abbreviations: Ant abd, anterolateral abdominal muscle; ASM, appendicular skeletal muscle mass; BMI, body mass index; FM, fat mass; HU, Hounsfield unit; LBM, lean body mass; Paraspinal, paraspinal muscle; Psoas, psoas major muscle; RKOA, radiographic knee osteoarthritis.

When data from males and females were analyzed separately, greater BMI and greater FM were associated with RKOA only in females, while greater LBM was associated with RKOA in both males and females after adjusting for age, height, and FM. In multivariate analyses, smaller paraspinal muscle area remained significantly associated with RKOA only in males, whereas in females, less paraspinal muscle attenuation was observed after adjusting for age. In the stepwise-regression analyses, when data from males and females were analyzed separately, paraspinal muscle attenuation, FM, and height were included in the final model for females, whereas paraspinal muscle area, LBM, and height were included in the final regression model for males.

\section{Association of body composition with knee pain and habitual exercise}

Subjective knee pain was reported by $39.8 \%$ of the males and $62.1 \%$ of the females. For both sexes, there were no significant differences in body composition between subjects with and without knee pain, although the prevalence of RKOA was two times greater in females with knee pain than in females without knee pain.

Habitual exercise was associated with RKOA in males. Females who habitually exercised had a lower \% body fat, larger psoas muscle and anterolateral muscle areas, greater muscle attenuation values, and greater ASM (appendicular LBM) than females who did not exercise regularly; in males, 
Table 3 Body composition characteristics of the study subjects stratified by knee pain or habitual exercise

\begin{tabular}{|c|c|c|c|c|c|c|c|c|}
\hline \multirow[t]{3}{*}{ Characteristics } & \multicolumn{4}{|l|}{ Knee pain } & \multicolumn{4}{|c|}{ Habitual exercise } \\
\hline & \multicolumn{2}{|l|}{ Male } & \multicolumn{2}{|l|}{ Female } & \multicolumn{2}{|l|}{ Male } & \multicolumn{2}{|l|}{ Female } \\
\hline & $(-)(n=80)$ & $(+)(n=53)$ & $(-)(n=47)$ & $(+)(n=77)$ & $(-)(n=8 I)$ & $(+)(n=63)$ & $(-)(n=97)$ & $(+)(n=38)$ \\
\hline RKOA (\%) & $17.5 \%$ & $22.6 \%$ & $17.0 \%$ & $35.1 \% *$ & $13.6 \%$ & $30.2 \% *$ & $27.1 \%$ & $30.8 \%$ \\
\hline Age & $62.8 \pm 14.3$ & $62.6 \pm 16.0$ & $66.4 \pm 11.3$ & $65.3 \pm 11.1$ & $61.2 \pm 15.5$ & $62.9 \pm 14.3$ & $66.0 \pm 11.6$ & $65.1 \pm 10.7$ \\
\hline Height (cm) & $168.8 \pm 7.3$ & $167.8 \pm 6.2$ & $153.3 \pm 6.5$ & $155.6 \pm 6.9$ & $168.6 \pm 6.7$ & $168.4 \pm 6.9$ & $154.7 \pm 6.6$ & $154.9 \pm 7.3$ \\
\hline BW (kg) & $68.1 \pm 10.2$ & $68.5 \pm 13.2$ & $52.5 \pm 10.0$ & $53.9 \pm 10.6$ & $67.8 \pm 9.5$ & $68.4 \pm 13.3$ & $53.7 \pm 11.1$ & $52.8 \pm 7.6$ \\
\hline $\mathrm{BMI}$ & $23.9 \pm 3.1$ & $24.2 \pm 3.8$ & $22.3 \pm 3.9$ & $22.3 \pm 4.2$ & $23.9 \pm 3.1$ & $24.0 \pm 3.8$ & $22.4 \pm 4.2$ & $22.1 \pm 3.3$ \\
\hline \multicolumn{9}{|l|}{ DEXA } \\
\hline$\%$ body fat & $25.3 \pm 6.7$ & $24.2 \pm 7.6$ & $32.5 \pm 7.9$ & $32.0 \pm 8.1$ & $25.3 \pm 7.0$ & $23.6 \pm 7.0$ & $33.0 \pm 8.0$ & $29.9 \pm 7.3 *$ \\
\hline $\mathrm{FM}(\mathrm{kg})$ & $17.7 \pm 6.6$ & $17.2 \pm 8.7$ & $17.6 \pm 7.2$ & $17.9 \pm 7.8$ & $17.6 \pm 6.6$ & $16.8 \pm 8.4$ & $18.4 \pm 7.9$ & $16.1 \pm 5.6$ \\
\hline LBM (kg) & $50.4 \pm 5.5$ & $51.3 \pm 6.8$ & $34.8 \pm 4.1$ & $36.1 \pm 4.6$ & $50.2 \pm 5.1$ & $51.6 \pm 6.8$ & $35.3 \pm 4.4$ & $36.7 \pm 4.5$ \\
\hline SMI & $7.3 \pm 0.7$ & $7.5 \pm 0.9$ & $5.7 \pm 0.6$ & $5.8 \pm 0.6$ & $7.4 \pm 0.8$ & $7.5 \pm 0.9$ & $5.7 \pm 0.6$ & $6.0 \pm 0.6 * *$ \\
\hline \multicolumn{9}{|l|}{ CT at umbilical level } \\
\hline Psoas $\left(\mathrm{cm}^{2}\right)$ & $23.1 \pm 5.5$ & $23.1 \pm 5.5$ & $12.4 \pm 3.0$ & $13.3 \pm 3.7$ & $22.5 \pm 5.8$ & $24.0 \pm 5.0$ & $12.5 \pm 3.5$ & $14.5 \pm 3.2^{*}$ \\
\hline Psoas HU & $48.8 \pm 4.0$ & $49.1 \pm 4.2$ & $44.6 \pm 4.1$ & $45.9 \pm 4.2$ & $48.5 \pm 4.1$ & $50.1 \pm 4.1 *$ & $44.8 \pm 4.2$ & $46.6 \pm 3.9 *$ \\
\hline Paraspinal $\left(\mathrm{cm}^{2}\right)$ & $36.1 \pm 9.0$ & $38.8 \pm 9.5$ & $23.4 \pm 6.7$ & $22.9 \pm 7.4$ & $36.9 \pm 8.8$ & $37.9 \pm 9.8$ & $22.8 \pm 7.5$ & $23.9 \pm 6.9$ \\
\hline Paraspinal HU & $46.5 \pm 6.5$ & $46.3 \pm 6.8$ & $37.4 \pm 6.2$ & $38.6 \pm 7.3$ & $46.2 \pm 7.2$ & $47.7 \pm 5.8$ & $37.6 \pm 7.1$ & $39.2 \pm 6.6$ \\
\hline Ant abd $\left(\mathrm{cm}^{2}\right)$ & $48.2 \pm 10.7$ & $50.5 \pm 11.9$ & $26.4 \pm 7.0$ & $27.7 \pm 8.7$ & $47.2 \pm 10.7$ & $51.9 \pm 11.7 *$ & $26.1 \pm 7.8$ & $30.2 \pm 8.6^{*}$ \\
\hline Ant abd HU & $43.9 \pm 6.1$ & $42.4 \pm 7.1$ & $36.9 \pm 7.2$ & $38.5 \pm 7.1$ & $43.2 \pm 7.4$ & $44.4 \pm 5.7$ & $36.8 \pm 7.3$ & $40.0 \pm 6.3^{*}$ \\
\hline $\operatorname{VAT}\left(\mathrm{cm}^{2}\right)$ & $110.2 \pm 54.3$ & $98.9 \pm 50.4$ & $70.5 \pm 43.0$ & $68.1 \pm 40.0$ & $106.2 \pm 55.0$ & $101.1 \pm 50.2$ & $71.4 \pm 43.2$ & $64.2 \pm 34.5$ \\
\hline
\end{tabular}

Notes: Habitual exercise was defined as exercise for at least 30 minutes per session, at least twice per week, and for at least I year. $* p<0.05$ and $* * p<0.0$ I vs. subjects without knee pain or habitual exercise by ANOVA; $(-)$, without knee pain or no habitual exercise; $(+)$, with knee pain or habitual exercise. Data presented as mean \pm SD unless otherwise indicated.

Abbreviations: ANOVA, analysis of variance; Ant abd, anterolateral abdominal muscle; BMI, body mass index; BW, body weight; CT, computed tomography; DEXA, dualenergy X-ray absorptiometry; FM, fat mass; HU, Hounsfield unit; LBM, lean body mass; Paraspinal, paraspinal muscle; Psoas, psoas major muscle; RKOA, radiographic knee osteoarthritis; SMI, skeletal muscle index; VAT, visceral adipose tissue.

these differences were less clear. In contrast, paraspinal muscle area and attenuation did not differ between male or female subjects who did or did not habitually exercise (Table 3).

\section{Discussion}

The results of this study showed an association between lower spinal muscle area/attenuation and RKOA, in contrast to the lack of associations between lower appendicular or total lean mass and RKOA. Rather, greater LBM was associated with RKOA after adjusting for age, height, and total adiposity, while total adiposity was associated with RKOA only in females and was no longer associated with RKOA after adjusting for age and BMI or lean mass.

High BMI is a well-known risk factor for both the onset and progression of knee OA.

In a European cohort study (the Rotterdam study), a high BMI at baseline was associated with the incidence and progression of knee OA. ${ }^{15}$ Recently, a systematic review and meta-analysis ${ }^{16}$ showed an association between knee extensor muscle weakness and increased risk of knee OA; this suggests that fat rather than lean mass underlies the association between greater BMI and knee OA. However, several reports have shown that both total/lean leg mass and FM were associated with RKOA. ${ }^{17-19}$ Moreover, it has been reported that greater leg lean mass was a more powerful predictor of RKOA than greater BMI. ${ }^{18}$

Segal et $\mathrm{al}^{20}$ reported that more thigh muscle mass was not associated with a lower risk of RKOA but that quadriceps strength was negatively associated with symptomatic knee OA and narrowing of the knee joint space. Although there is a strong positive association between muscle mass and strength, greater muscular mass does not necessarily mean greater muscular strength; the so-called low-quality muscle, common in elderly or sedentary individuals, may not be strong enough to alleviate the burden on the knee joints and may (similar to FM) even impose a higher mechanical burden on the knee.

The novel finding in our study is that, unlike total or lean leg mass, greater abdominal muscle area, especially paraspinal muscle area, is associated with less risk of RKOA. We do not have any mechanistic data; however, the paraspinal muscles are the only extensors of the trunk, and their stabilizing function could be of particular importance for balance and mobility in older adults, especially because people in this age group often suffer from a flexed (i.e., kyphotic) posture. Agerelated kyphosis due to small and weak paraspinal muscles may increase the mechanical burden on the knee joint. Since this is a cross-sectional study, it is important to consider that a kyphotic posture could be the result of knee pain due to 
RKOA rather than the cause of RKOA. However, we found no associations between knee pain and abdominal muscle parameters, suggesting that the smaller paraspinal muscles in subjects with RKOA were not merely a consequence of knee pain.

A recent 15-year longitudinal observational study ${ }^{21}$ showed that the area and fatty infiltration of the paraspinal muscles were dependent on age and were not associated with physical activity level or degree or history of lumber back pain among typical Finnish males. In fact, familial aggregation (the combined effects of genes and a shared early environment) has been reported to be the strongest predictor of paraspinal muscle parameters. ${ }^{22}$ The lumbar paraspinal muscles help to stabilize the spine and maintain proper posture; thus, these muscles are used in any movement against gravity in daily life. Other abdominal muscles, including the anterolateral abdominal and psoas muscles, are more often used during the events such as heavy exercise or intentional brisk walking. In the current study, the paraspinal muscles were the trunk muscles that were least associated with habitual exercise. However, although no relationships were observed between habitual exercise and paraspinal muscle morphology, most of our study subjects performed aerobic exercise, which may place less stress on the lumbar extensor muscles than resistance exercise. An earlier case-control study showed that 70-year-old males with a history of aerobic exercise had similar arm and leg muscle areas as age-matched sedentary controls, whereas males who had resistance training had muscle area comparable with a group of sedentary 28-year-old subjects; this suggests that resistance training is key to maintaining muscle mass. ${ }^{23}$ Therefore, resistance exercises designed to stimulate the lumbar extensor muscles may be a promising treatment option for knee OA.

Muscle CT attenuation is a marker of fatty infiltration of the muscle, and lower muscle attenuation is associated with less muscular strength, independent of muscle area, in older people. ${ }^{14}$ Using magnetic resonance imaging (MRI), Kumar et $\mathrm{al}^{24}$ also reported more quadriceps intramuscular fat and less quadriceps strength in people with knee OA, without concomitant changes in quadriceps area. In the current study, lower paraspinal muscle attenuation values were also associated with RKOA, especially in females.

The effect of FM on knee OA was more apparent in females than in males, and this is consistent with previous observations. ${ }^{15,25}$ A recent study of Asian adults aged between 20 and 90 years $^{25}$ found that advancing age was associated with knee OA in both males and females, while greater FM was associated with knee OA only in females. The reason for the observed sex difference is unclear; however, females have more fat and less muscle and are more prone to gaining fat in both adipose tissue and muscle, which worsens muscle quality. In a large-scale population-based cohort study of older Japanese adults (ROAD study), sitting on a chair was found to be a significant protective factor against RKOA, while standing, walking, climbing, and heavy lifting were associated with RKOA. ${ }^{26}$ In the current study, habitual exercise negatively affected RKOA only in males. It can be hypothesized that habitual exercise (which was almost exclusively walking in our study population) was not associated with RKOA in females because it prevents fat gain and fatty infiltration of the muscles.

The results of this study should be interpreted within the context of its limitations. Since this was a cross-sectional study, we cannot draw any conclusions about the causal or directional nature of the associations of muscle mass and quality with RKOA; although considering the lack of association between abdominal muscles and knee pain, it is unlikely that the observed association between the paraspinal muscles and RKOA was merely a consequence of knee pain. Moreover, although thigh/quadriceps muscle mass/strength is a well-known risk/protective factor for RKOA, we did not assess this directly. However, we did use DEXA to assess body composition and ASM, which is almost reflective of leg lean mass. We also did not assess abdominal muscle strength; however, muscle CT attenuation is a good index of muscular quality and strength. Our subjects were limited to medical checkup examinees and were more likely to be motivated for healthier lifestyle, and therefore, the selection bias also needs to be considered to generalize the findings. Finally, our definition of habitual exercise was not following the actual physical activity recommendations on a health perspective worldwide. ${ }^{27}$

Despite these limitations, our current study adds to the current body of knowledge on the role of the size/quality of the paraspinal muscles in knee OA. The lack of a protective effect of total/appendicular lean mass also strongly suggests the importance of muscular size/quality rather than muscle quantity. So far, clinical recommendations to alleviate knee pain and slow the progression of knee OA consist of weight loss and resistance training of the quadriceps. Based on our observations, resistance exercise of the trunk extensor muscles or posture training may be other promising options for the treatment of knee OA. These exercises also have the advantage of placing no direct mechanical stress on the knee joints. 


\section{Conclusion}

We observed that smaller paraspinal muscles were associated with RKOA and that total/appendicular lean mass did not protect against knee OA. This suggests the potential promising option of resistance training for the paraspinal muscles to alleviate knee OA.

\section{Acknowledgments}

We thank the staff of the radiology department for their support with the CT and DEXA measurements. We are also indebted to the staff of the Institute for Integrated Sports Medicine for their assistance with data collection. This work was supported by JSPS KAKENHI 15K01726.

\section{Disclosure}

The authors report no conflicts of interest in this work.

\section{References}

1. Fransen M, Bridgett L, March L, Hoy D, Penserga E, Brooks P. The epidemiology of osteoarthritis in Asia. Int J Rheum Dis. 2011;14(2): 113-121.

2. Yoshimura N, Muraki S, Oka H, et al. Prevalence of knee osteoarthritis, lumbar spondylosis, and osteoporosis in Japanese men and women: the research on osteoarthritis/osteoporosis against disability study. $J$ Bone Miner Metab. 2009;27(5):620-628.

3. Blagojevic M, Jinks C, Jeffery A, Jordan KP. Risk factors for onset of osteoarthritis of the knee in older adults: a systematic review and meta-analysis. Osteoarthritis Cartilage. 2010;18(1):24-33.

4. Granacher U, Gollhofer A, Hortobagyi T, Kressig RW, Muehlbauer T. The importance of trunk muscle strength for balance, functional performance, and fall prevention in seniors: a systematic review. Sports Med. 2013;43(7):627-641.

5. Hodges PW, Richardson CA. Contraction of the abdominal muscles associated with movement of the lower limb. Phys Ther. 1997;77(2):132-142; discussion 142-134.

6. Willson JD, Dougherty CP, Ireland ML, Davis IM. Core stability and its relationship to lower extremity function and injury. $J$ Am Acad Orthop Surg. 2005;13(5):316-325.

7. Earl JE, Hoch AZ. A proximal strengthening program improves pain, function, and biomechanics in women with patellofemoral pain syndrome. Am J Sports Med. 2011;39(1):154-163.

8. Kellgren JH, Lawrence JS. Radiological assessment of osteo-arthrosis. Ann Rheum Dis. 1957;16(4):494-502.

9. Culvenor AG, Engen CN, Oiestad BE, Engebretsen L, Risberg MA. Defining the presence of radiographic knee osteoarthritis: a comparison between the Kellgren and Lawrence system and OARSI atlas criteria. Knee Surg Sports Traumatol Arthrosc. 2015;23(12):3532-3539.

10. Schiphof D, Boers M, Bierma-Zeinstra SM. Differences in descriptions of Kellgren and Lawrence grades of knee osteoarthritis. Ann Rheum Dis. 2008;67(7):1034-1036.

11. Abellan van Kan G, Houles M, Vellas B. Identifying sarcopenia. Curr Opin Clin Nutr Metab Care. 2012;15(5):436-441.
12. Schweitzer L, Geisler C, Pourhassan M, et al. What is the best reference site for a single MRI slice to assess whole-body skeletal muscle and adipose tissue volumes in healthy adults? Am J Clin Nutr. 2015;102(1):58-65.

13. Goodpaster BH, Kelley DE, Thaete FL, He J, Ross R. Skeletal muscle attenuation determined by computed tomography is associated with skeletal muscle lipid content. J Appl Physiol. 2000;89(1): 104-110.

14. Goodpaster BH, Carlson CL, Visser M, et al. Attenuation of skeletal muscle and strength in the elderly: the Health ABC study. J Appl Physiol. 2001;90(6):2157-2165.

15. Reijman M, Pols HA, Bergink AP, et al. Body mass index associated with onset and progression of osteoarthritis of the knee but not of the hip: the Rotterdam study. Ann Rheum Dis. 2007;66(2): $158-162$.

16. Oiestad BE, Juhl CB, Eitzen I, Thorlund JB. Knee extensor muscle weakness is a risk factor for development of knee osteoarthritis. A systematic review and meta-analysis. Osteoarthritis Cartilage. 2015;23(2):171-177.

17. Abbate LM, Stevens J, Schwartz TA, Renner JB, Helmick CG, Jordan JM. Anthropometric measures, body composition, body fat distribution, and knee osteoarthritis in women. Obesity (Silver Spring). 2006;14(7):1274-1281.

18. Blumenfeld O, Williams FM, Hart DJ, Arden NK, Spector TD, Livshits G. Lower limbs composition and radiographic knee osteoarthritis (RKOA) in Chingford sample - a longitudinal study. Arch Gerontol Geriatr. 2013;56(1):148-154.

19. Sowers MF, Yosef M, Jamadar D, Jacobson J, Karvonen-Gutierrez C, Jaffe M. BMI vs. body composition and radiographically defined osteoarthritis of the knee in women: a 4-year follow-up study. Osteoarthritis Cartilage. 2008;16(3):367-372.

20. Segal NA, Findlay C, Wang K, Torner JC, Nevitt MC. The longitudinal relationship between thigh muscle mass and the development of knee osteoarthritis. Osteoarthritis Cartilage. 2012;20(12) 1534-1540.

21. Fortin M, Macedo LG. Multifidus and paraspinal muscle group cross-sectional areas of patients with low back pain and control patients: a systematic review with a focus on blinding. Phys Ther. 2013;93(7):873-888.

22. Gibbons LE, Videman T, Battie MC, Kaprio J. Determinants of paraspinal muscle cross-sectional area in male monozygotic twins. Phys Ther. 1998;78(6):602-610; discussion 611-602.

23. Klitgaard H, Mantoni M, Schiaffino S, et al. Function, morphology and protein expression of ageing skeletal muscle: a cross-sectional study of elderly men with different training backgrounds. Acta Physiol Scand. 1990;140(1):41-54.

24. Kumar D, Karampinos DC, MacLeod TD, et al. Quadriceps intramuscular fat fraction rather than muscle size is associated with knee osteoarthritis. Osteoarthritis Cartilage. 2014;22(2):226-234.

25. Ho-Pham LT, Lai TQ, Mai LD, Doan MC, Nguyen TV. Body composition in individuals with asymptomatic osteoarthritis of the knee. Calcif Tissue Int. 2016;98(2):165-171.

26. Muraki S, Akune T, Oka H, et al. Association of occupational activity with radiographic knee osteoarthritis and lumbar spondylosis in elderly patients of population-based cohorts: a large-scale population-based study. Arthritis Rheum. 2009;61(6):779-786.

27. Riebe D, Franklin BA, Thompson PD, et al. Updating ACSM's recommendations for exercise preparticipation health screening. Med Sci Sports Exerc. 2015;47(11):2473-2479. 


\section{Publish your work in this journal}

Open Access Rheumatology: Research and Reviews is an international, peerreviewed, open access journal publishing original research, reports, editorials, reviews and commentaries on all aspects of clinical and experimental rheumatology in the clinic and laboratory including the following topics: Pathology, pathophysiology of rheumatological diseases; Investigation, treatment and management of rheumatological diseases; Clinical trials and novel pharmacologi$\mathrm{cal}$ approaches for the treatment of rheumatological disorders. The manuscrip management system is completely online and includes a very quick and fair peer-review system, which is all easy to use. Visit http://www.dovepress.com testimonials.php to read real quotes from published authors. 